\title{
Thyroid Hormone Resistant Syndrome
}

\author{
Karo Gyurjian, OMS IV ${ }^{1}$; Vishwanath Venketaraman, PhD $^{2}$ \\ ${ }^{1}$ College of Osteopathic Medicine of the Pacific, Pomona, CA \\ ${ }^{2}$ Western University of Health Sciences, Pomona, CA
}

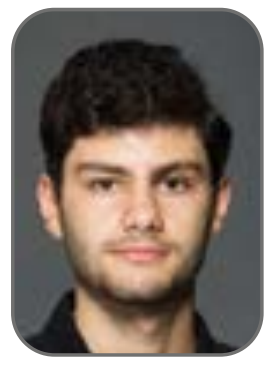

Karo Gyurjian, OMS IV

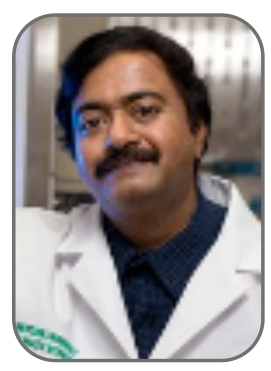

Vishwanath Venketaraman, PhD

\section{CORRESPONDENCE:}

Vishwanath Venketaraman, PhD vvenketaraman@westernu.edu

DOI: $10.38206 / 130101$

\section{KEYWORDS:}

Elevated Thyroid Hormone Levels, Endocrinology, THR, Thyroid Hormone Resistance

\section{ABSTRACT}

Thyroid hormone resistance (THR), also known as resistance to thyroid hormone (RTH), is an inherited condition characterized by reduced end-organ responsiveness to thyroid hormone, caused by mutations in the thyroid hormone receptor gene. Patients typically present with elevated thyroid hormone levels (T3 and T4) with normal, or slightly elevated thyroid stimulating hormone (TSH) levels. ${ }^{1}$ In a majority of cases, the disease is caused by a mutation in the thyroid receptor beta (TR-beta) gene. Patients can present with signs and symptoms of hypothyroidism or hyperthyroidism or can be asymptomatic. We present a case of a 16-year-old male who was referred for endocrinologic evaluation after abnormal findings in the thyroid function panel.

\section{INTRODUCTION}

Resistance to thyroid hormone (RTH) is an autosomal dominant disorder characterized by reduced end-organ sensitivity to thyroid hormone, leading to elevated levels of T4 and T3 accompanied by normal or slightly elevated levels of $\mathrm{TSH}$. The syndrome has a prevalence of about 1 in 40,000 live births, occurring with equal frequencies in both sexes. ${ }^{2}$ The overwhelming majority of cases are caused by mutations in the thyroid receptor -beta gene, interfering with the physiologic function of the thyroid receptor. ${ }^{3}$ Thyroid hormone has a variety of functions on many different tissue types and organs in the body. The severity of hormonal resistance varies among different tissue types, probably due to the variable expression of thyroid receptor throughout different organs. ${ }^{4}$
Due to the decreased sensitivity to thyroid hormone, the elevated levels of T4 and T3 fail to downregulate the production of TSH from the anterior pituitary gland, as demonstrated by the normal or elevated TSH lab values. Patients can be asymptomatic, or can present with either hypothyroid (growth retardation, delayed bone maturation, learning disabilities, sensorineural deafness) or hyperthyroid (tachycardia, hyperactivity, increased basal metabolic rate) features. ${ }^{5}$ With labs that mimic hyperthyroidism and a nonspecific clinical presentation, patients can be misdiagnosed and even unnecessarily treated with invasive techniques (radioactive iodine ablation) that can further exacerbate the underlying 'hypothyroidism'. Treatment is not indicated in a majority of cases as the hyposensitivity to thyroid hormone is 
adequately compensated by the increased thyroid hormone levels. ${ }^{6}$ Patients who develop a large goiter due to increased TSH levels can be treated with highdose triiodothyronine to help regress the goiter.?

In the following case, we will discuss a patient who presented with poor weight gain, learning disabilities and bilateral sensorineural hearing loss. The patients' laboratory workup revealed elevated thyroid hormone levels accompanied by normal TSH levels without any overt signs of hyperthyroidism or hypothyroidism.

\section{CASE:}

A 16-year-old male patient was diagnosed with Resistance to Thyroid Hormone at the age of four-and-a half years old when thyroid function tests revealed elevated total T4, free T4, T3 and normal TSH levels, without any obvious clinical signs of hyper- or hypothyroidism. The patient initially presented with poor weight gain and low $\mathrm{BMI}$ (his most recent labs reveal his height and weight to be $<1$ percentile and his $\mathrm{BMI}$ at 4 th percentile). At that time, the patient's bone age was determined to be six years-old (when his chronological age was 4.5 years-old). This was thought to be secondary to the relatively increased sensitivity to thyroid hormone in certain tissues, such as bone. Both his parents, and siblings had thyroid function tests completed, and tested normal.

\section{Past Medical History}

The patient was born at 37 weeks gestation, with no complications, no history of gestational diabetes, intrauterine growth restrictions (IUGR) or small for gestational age (SGA). The patient was diagnosed with bilateral sensorineural hearing loss, for which he uses hearing aids. He has learning disabilities and is currently in special education. Earlier on, during childhood development, there was a concern for possible attention deficit disorder (ADD) for this patient, ${ }^{8}$ but this is no longer a concern as he does not demonstrate signs of the disorder. He has no other prior hospitalizations or surgeries. The patient has seasonal allergies, and he is allergic to Amoxicillin (develops a rash upon exposure).

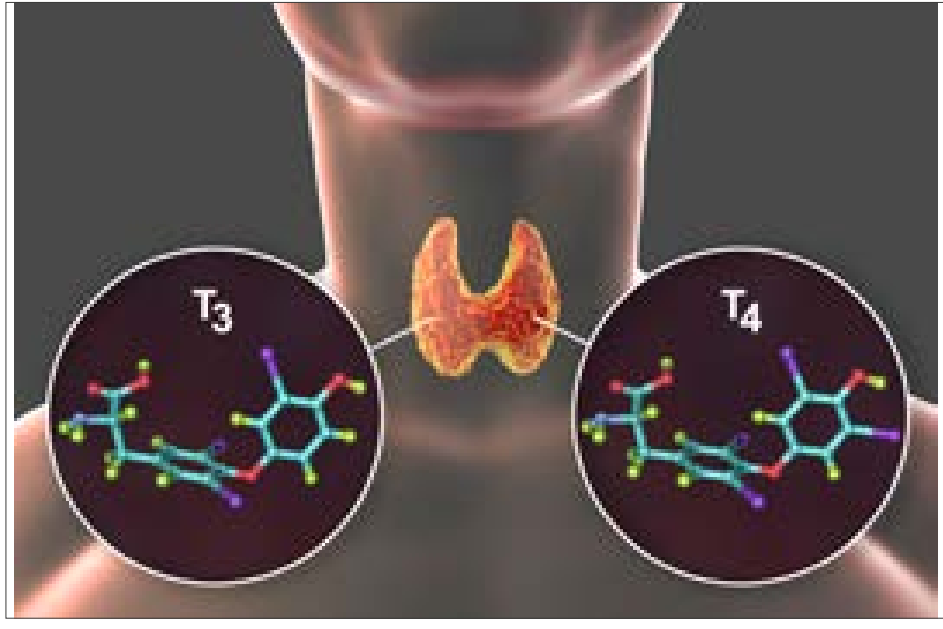

\section{Family history}

Family history is negative for thyroid hormone resistance or any other thyroid pathology, as confirmed by normal thyroid function tests completed by both parents and his siblings. The mother's height is $4^{\prime} 8^{\prime \prime}$ and father's height is $5^{\prime} 2 "$, therefore, the patient's short stature is within his mid-parental target height. The patient was, however, growing above his genetic potential but due to his advanced bone age, has obtained an adult height of $5^{\prime} 1.58^{\prime \prime}$ at the age of 16 .

\section{Physical Exam}

On physical exam, he has a blood pressure of 117/57, a pulse of 67 , temperature of $37^{\circ} \mathrm{C}$, weight of $92 \mathrm{lb} 13 \mathrm{oz}$ $(<1$ percentile), and a height of $156.4 \mathrm{~cm}(<1$ percentile). $\mathrm{His} \mathrm{BMI}$ is at the 4th percentile (Figure 1). The patient appears in no acute distress, with no dysmorphic features, but is thin. He demonstrates delayed, slow responses but is interacting well and answering appropriately for the most part. Examination of the eyes shows mild bilateral proptosis. No thyromegaly or thyroid nodules are noted on examination of the neck. Neurologic exam is non-focal. Patellar and brachioradialis DTRs are 2+ bilaterally. The patient also has bilateral sensorineural hearing loss and uses hearing aids. 


\section{LABORATORY STUDIES}

Laboratory results consistently demonstrate normal TSH but elevated Total T4, Free T4 and Total T3. These findings are indicative of end-organ resistance at the anterior pituitary gland (Table 1). Based on the negative feedback principles of functional physiology, we expect elevated values of T4 and T3 to suppress TSH production. The normal values of TSH indicates lack of negative feedback, due to thyroid hormone receptor polymorphism which inhibits physiologic hormone signal transduction.

\section{SNP microarray}

46XY with normal copy number. High density of short contiguous regions of homozygosity. Per genetics: this suggests an increase in autosomal recessive allele risk.

\section{Impression and plan}

The patient is a 16-year-old male who presents with growth delay, intellectual disabilities, bilateral sensorineural hearing loss and lab values demonstrating consistently elevated thyroid hormone with normal TSH levels. He is clinically euthyroid. Although no further genetic analyses were done, his clinical presentation and lab findings are consistent with thyroid hormone resistance, most likely secondary to a mutation in the thyroid receptor-beta gene. He does not have a family history of THR, which indicates either a de novo mutation or possibly, autosomal recessive transmission.

The primary differential diagnosis in this case would be a TSH-secreting pituitary adenoma, given his elevated thyroid hormone levels and unsuppressed TSH. However, the patient doesn't show signs of a pituitary adenoma as he does not have any mass-effect findings (bitemporal hemianopsia), thyromegaly or other hormonal derangements (pituitary adenomas co-secrete). Furthermore, the patient does not demonstrate the classic signs of hyperthyroidism that would be present in a TSH-secreting pituitary adenoma (palpitations, tachycardia, unintentional weight loss, heat intolerance, insomnia, restlessness).

Since the patient is clinically stable and his developmental delay is not profound, he is currently not undergoing any treatment with thyroid hormone. Certain cases of THR require supraphysiologic doses of levothyroxine to overcome end-organ resistance. The patient is currently under the care of endocrinology, following up with yearly laboratory studies. He is also under the care of pediatrics, for continued management of weight trends.

\section{DISCUSSION}

We describe a case of a 16-year-old male with poor weight gain, bilateral sensorineural hearing loss and intellectual disabilities. Further laboratory studies revealed elevated thyroid hormone levels in the setting of normal TSH values indicating a failure to suppress TSH production. The clinical presentation and laboratory findings are indicative of THR. The syndrome is characterized by decreased peripheral and pituitary sensitivity to thyroid hormone due to mutations in the thyroid receptor-beta (TRbeta) gene located on chromosome 3 .

THR is detected in about 1/40,000 live births with an overwhelming majority demonstrating autosomal dominant inheritance pattern. It is particularly interesting to note, that in our case, there is no pertinent family history of thyroid hormone resistance or any other thyroid hormone pathology. Lack of positive family history combined with his SNP microarray assay which demonstrates a high density of short contiguous regions of homozygosity, the patient in this case likely developed the syndrome due to an autosomal recessive inheritance ${ }^{9}$ or a de novo mutation in the TR-beta gene. 
Common clinical findings encountered with THR include goiter (65-95\% of cases), hyperactivity (3368\%) and tachycardia (33-75\%). ${ }^{10}$ These clinical signs combined with the laboratory findings of elevated thyroid hormone levels often results in the erroneous diagnosis of hyperthyroidism. Patients are subsequently subjected to ablative or antithyroid treatment to reduce thyroid hormone levels, which can further exacerbate an underlying 'hypothyroid' state. It is therefore critical for physicians to recognize the presentation of THR in order to carry out proper management of the syndrome.

The patient in our case did not demonstrate any of the classic findings of hyperthyroidism (goiter, hyperactivity, tachycardia). Instead, he presented with findings such as poor weight gain, low BMI, bilateral sensorineural hearing loss, and learning disabilities. These findings have been reported in patients with thyroid hormone resistance, but at reduced rates. The poor weight gain and low BMI can be explained by the elevated thyroid hormone levels, since the degree of insensitivity to thyroid hormone varies throughout different body tissues. Studies have demonstrated that patients with THR have increased muscle-derived resting energy expenditure as a consequence of thyroid hormone-mediated mitochondrial uncoupling. ${ }^{11}$

The patient's bilateral sensorineural hearing loss can also be explained by his THR. Studies have demonstrated the importance of TR-beta in the development of the inner ear. Studies on mice have shown that the absence of TR-beta-1 gene is sufficient to cause hearing loss. ${ }^{12}$

There is a high prevalence of learning disabilities in patients with thyroid hormone resistance. In a study carried out to describe the genetic and clinical features of thyroid hormone resistance, $38 \%$ of the subjects were found to have an $1 \mathrm{Q}<85$ and $35 \%$ had speech impediment. Thyroid hormones play a critical role in brain development with their influence on neurogenesis, neuronal migration, neuronal and glial cell differentiation, and synaptogenesis. The actions of thyroid hormones are carried out through hormonal interaction with intranuclear receptors, leading to regulation of gene expression. Studies conducted on mice expressing a mutant TR-beta-1 gene demonstrated properties similar to those seen with severe hypothyroidism, such as neuromotor disability. ${ }^{13}$
Treatment is not required in a majority of cases of THR as the elevated thyroid hormone levels tend to compensate for the decreased end-organ sensitivity. However, certain groups of patients do require treatment to manage their symptoms. Patients who have been erroneously treated for hyperthyroidism with ablative therapy require thyroid hormone supplementation to decrease TSH levels to normal. These patients have a decreased thyroid hormone reserve, requiring supplementation. Certain patients, however, develop goiters and thyromegaly. Regression of large goiters has been successfully achieved with the administration of a single high dose of Liothyronine (L-T3) given every other day. ${ }^{?}$

In certain patients with THR, peripheral tissues may be relatively more resistant than thyrotropes in the anterior pituitary. This can lead to inadequate compensation for the hormonal resistance in these tissues. In these cases, T4 administration beyond stabilization of TSH levels is required. The adequate dose needs to be determined on an individual basis by regularly assessing the patient's response to thyroid hormone. This should be carried out with regular assessment of growth, bone maturation, and mental development over long-term care. Since our patient is clinically euthyroid, has obtained mature adult height and his developmental delay is stable (he is in 11th grade, special education), the endocrinologist has decided against treatment with thyroid hormone. The patient is under the care and management of endocrinology and pediatrics with regular laboratory studies and assessment of weight changes. He has been showing improved signs of increasing BMI percentiles.

As depicted in the growth chart (Figure 1), the patient consistently falls at or below the 5th percentile in the BMI Growth charts. The low BMI can be explained by the increased levels of circulating thyroid hormones. The patient's most recent weight was 92lbs and $130 z$. Studies have demonstrated that patients with THR have increased muscle-derived resting energy expenditure as a consequence of thyroid hormone-mediated mitochondrial uncoupling. The patient's most recent height was measured at $156.4 \mathrm{~cm}$. The short stature of the patient can be explained by the average height of his parents (mother, $4^{\prime} 8^{\prime \prime}$ and father, $5^{\prime} 2^{\prime \prime}$ ) and also, due to his increased rate of bone maturation, secondary to thyroid hormone. 


\section{AUTHOR DISCLOSURES:}

No relevant financial affiliations or conflicts of interest.

\section{REFERENCES:}

1. Refetoff, S. "The Syndromes of Resistance to Thyroid Hormone." Endocrine Reviews, vol. 14, no. 3, 1993, pp. 348-399., doi:10.1210/er.14.3.348.

2. Lafranchi, Stephen H, et al. "Followup of Newborns with Elevated Screening T4 Concentrations." The Journal of Pediatrics, vol. 143, no. 3, 2003, pp. 296-301., doi:10.1067/ s0022-3476(03)00184-7.

3. Olateju, Tolulope $O$, and Mark P J Vanderpump. "Thyroid Hormone Resistance." Annals of Clinical Biochemistry, vol. 43, no. 6, 2006, pp. 431-440., doi:10.1258/000456306778904678.

4. Flamant, Frédéric, and Jacques Samarut. "Thyroid Hormone Receptors: Lessons from Knockout and Knock-in Mutant Mice." Trends in Endocrinology \& Metabolism, vol. 14, no. 2, 2003, pp. 85-90., doi:10.1016/s1043-2760(02)00043-7.

5. Beck-Peccoz, Paolo, and V. Krishna K. Chatterjee. "The Variable Clinical Phenotype in Thyroid Hormone Resistance Syndrome." Thyroid, vol. 4, no. 2, 1994, pp. 225-232., doi:10.1089/thy.1994.4.225.

6. Weiss, R. E. "Treatment of Resistance to Thyroid Hormone--Primum Non Nocere." Journal of Clinical Endocrinology \& Metabolism, vol. 84, no. 2, 1999, pp. 401-404., doi:10.1210/jc.84.2.401.

7. Anselmo, João, and Samuel Refetoff. "Regression of a Large Goiter in a Patient with Resistance to Thyroid Hormone by Every Other Day Treatment with Triiodothyronine." Thyroid, vol. 14, no. 1, 2004, pp. 71-74., doi:10.1089/105072504322783876.

8. Hauser, Peter, et al. "Attention Deficit-Hyperactivity Disorder in People with Generalized Resistance to Thyroid Hormone." New England Journal of Medicine, vol. 328, no. 14, 1993, pp. 997-1001., doi:10.1056/nejm199304083281403.

9. Refetoff, Samuel, et al. "Impaired Sensitivity to Thyroid Hormone." UpToDate, 24 May 2017, www.uptodate.com/ contents/impaired-sensitivity-to-thyroid-hormone.

FIGURE 1.
Patient's Trends on BMI Growth Chart

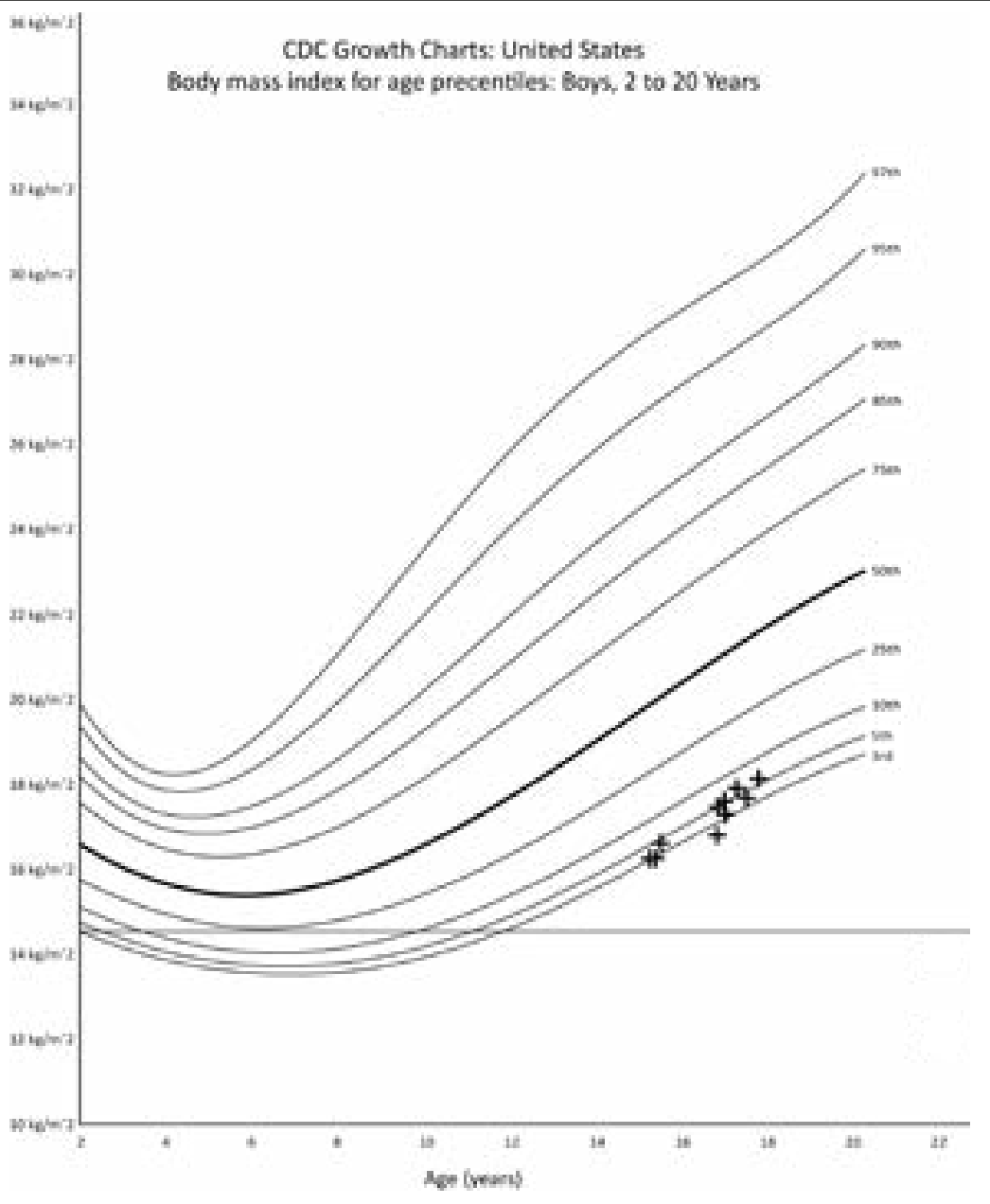

10. Brucker-Davis, Francoise. "Genetic and Clinical Features of 42 Kindreds with Resistance to Thyroid Hormone: The National Institutes of Health Prospective Study." Annals of Internal Medicine, vol. 123, no. 8, 1995, p. 572., doi:10.7326/0003-4819-123-8-199510150-00002.

11. Mitchell, Catherine S., et al. "Resistance to Thyroid Hormone Is Associated with Raised Energy Expenditure, Muscle Mitochondrial Uncoupling, and Hyperphagia." Journal of Clinical Investigation, vol. 120, no. 4, 2010, pp. 1345-1354., doi:10.1172/jci38793.

12. Abel, E. Dale, et al. "Divergent Roles for Thyroid Hormone Receptor B Isoforms in the Endocrine Axis and Auditory System." Journal of Clinical Investigation, vol. 104, no. 3, 1999, pp. 291-300., doi:10.1172/jci6397.

13. Bernal, J. "Thyroid Hormones and Brain Development." Hormones, Brain and Behavior, 2009, pp. 20052034., doi:10.1016/b978-008088783-8.00063-2. 